Review Article

\title{
Association of passive smoking and lung cancer: Is there substantial evidence to prove the causation?
}

\author{
Sudhir Kumar Ambati* \\ Department of Epidemiology, University of Medicine and Health Science, St. Kitts and Nevis, Netherlands Antilles \\ Received: 15 March 2021 \\ Revised: 29 March 2021 \\ Accepted: 30 March 2021

\section{*Correspondence:} \\ Dr. Sudhir Kumar Ambati, \\ E-mail: sudhirambati@gmail.com \\ Copyright: (C) the author(s), publisher and licensee Medip Academy. This is an open-access article distributed under \\ the terms of the Creative Commons Attribution Non-Commercial License, which permits unrestricted non-commercial \\ use, distribution, and reproduction in any medium, provided the original work is properly cited.
}

\begin{abstract}
Cigarette smoking plays a crucial role in the development of lung cancer. Tobacco smoke positively influences lung cancer development in both smokers and never or non-smokers. The influence of passive smoke on lung cancer development is associated with toxic substances that cause chronic inflammation in the respiratory systems. Frequent or high exposure to second-hand or environmental tobacco smoke causes respiratory infections that impair lung function and triggers lung cancer development through cell damage, mitosis, and apoptosis. The toxic substances found in second-hand smoke, including carcinogens, ammonia, carbon monoxide, and nitrosamines, have a significant association with squamous cell carcinoma, small cell lung carcinoma, large cell carcinoma, and adenocarcinoma. Although some previously conducted studies showed no significant association between passive smoking and lung cancer, recent studies provide substantial evidence between their associations. Thus, exposure to passive smoke increases the risks of lung cancer.
\end{abstract}

Keywords: Lung cancer, Small cell lung carcinoma, Large cell carcinoma, Adenocarcinoma, Squamous cell carcinoma, Passive smoking, Environmental tobacco smoke, Second-hand smoke

\section{INTRODUCTION}

Cigarette smoking is common for both the adult and young population, with an estimated one-third of the adult population using tobacco globally and $88 \%$ of this population coming from low-income countries. ${ }^{1}$ The increasing incidence and mortality of tobacco smoking have immediate effects on human health. Those who smoke cigarettes are more susceptible to tobacco-related diseases, including respiratory infections, allergies, ear infections, enhanced risks for asthma, and impaired growth of lungs. ${ }^{2}$ Existing clinical evidence supports the significant contributions of cigarette smoking to the etiology of lung cancer. ${ }^{3}$ Lung cancer remains the main cause of cancer-related deaths among both men and women globally. The prevalence and mortality of lung cancer are highly associated with the patterns of cigarette smoking. ${ }^{4}$ It is evident that cigarette use is highly respon- sible for cancer-related deaths, and it has been established that smoking accounts for $87 \%$ of lung cancer deaths compared to $30 \%$ of all cancer deaths. Among these lungcancer cases and deaths, both men and women smokers account for $29 \%$ and $26 \%$ cases, respectively. ${ }^{1}$

Passive smoking entails a combination of two types of smoke from the burning tobacco, which are classified as side-stream smoke and mainstream smoke. The sidestream smoke originates from the end of the lighted sources, such as the cigar, pipe, or cigarette itself, which comprises very small particles rich in carcinogens and can easily enter the cells. ${ }^{1}$ The mainstream smoke is mainly exhaled by the tobacco smoker and makes its way easily to the cells when inhaled by another person. ${ }^{1}$ This present research, therefore, aims to investigate the association between passive smoking and lung cancer. The research question to address is: Is there substantial evidence to 
prove the association between passive smoking and lung cancer?

\section{WHAT IS PASSIVE SMOKING?}

Passive smoking, environmental or second-hand smoke, entails a mixture of the smoke exhaled by the cigarette user and the smoke from the burning tip or the end of the cigarette. When a person inhales this type of smoke, it is termed passive smoking since it occurs through partial inhalation of the air from the smoker. ${ }^{5}$ People who do not smoke are frequently exposed to passive smoking when in contact with a nearby smoker, making them more prone to health hazards. Non-smokers are exposed to passive smoking, mainly in public places, including offices, cars, schools, shopping centres, public transportations, restaurants, and parks. ${ }^{5}$ Besides, when parents smoke at home, they expose their children and other people to passive smoking, and these chidden are highly sensitive to toxins in the smoke. These toxins include ammonia, carbon monoxide, and nitrosamines, making people highly susceptible to health hazards, including cancer. Passive smoking causes various health problems among children and adults, and the most commonly known health issues are respiratory infections, coronary heart diseases, lung cancer, and stroke. ${ }^{6}$

\section{ASSOCIATION BETWEEN PASSIVE SMOKING AND LUNG CANCER}

Passive smoke is blamed for total cases of lung cancer. The association is known to be dose-dependent, and small cell lung cancer is the main histological type that has a strong association with passive smoking. ${ }^{11}$ Second-hand smoking exposure to environmental tobacco smoke at home and work plays a key role in the pathogenesis of lung cancer among non-smokers. ${ }^{16,17}$ The second-hand smoking at home is considered to have a greater impact on the development of lung cancer than exposure to second-hand smoking in the office. Exposure to such smoke during childhood enhances the risks of lung cancer in adulthood. Such an increase is because of the greater duration and intensity of smoke exposure at home than in other places. ${ }^{14}$ As a consequence, children and other people in contact with smokers at home are at higher risks of developing lung cancer than those far from such people. The possibility of non-smokers having lung cancer due to passive smoking is predicted to be increased by $25 \%$, and the percentage is expected to increase in the future dramatically. ${ }^{15}$ Existing studies also support the association between passive smoking and lung cancer.

\section{DISCUSSION}

It is well established that passive smoking is the key cause of lung cancer. A previously conducted systematic review that investigated the occurrence of lung cancer among nonsmokers in China supported that passive smoking increases the risk and enhances the onset of lung cancer. ${ }^{7}$ The biological plausibility for such a relationship is that the toxins or toxic substances and carcinogens present on sidestream and mainstream smoke exhaled by smokers facilitate the development of lung cancer. ${ }^{7}$ Both childhood and adulthood exposure to environmental tobacco smoke increases the risks for lung cancer across genders. A NordTrondelag Health Survey (HUNT) prospective cohort study that examined the impacts of passive smoking in both childhood and adulthood on the occurrences of lung cancer and histological types showed a strong association. ${ }^{8}$ The HUNT study revealed that passive smoking during childhood only, adulthood only, and during both periods was highly associated with lung cancer. The findings established that there was a 50-52\% increased risk for lung cancer after adjusting for active smoking and other related confounders. ${ }^{8}$ The study showed increased risks for histological types of lung cancer, such as squamous cell carcinoma and small cell lung cancer, but a weaker association for adenocarcinoma. ${ }^{8}$ From such findings, lung cancer in people who never smoke could be attributed to passive smoking or high exposure to tobacco smoke from smokers. Household exposure to environmental tobacco smoke increases the individual risk for lung cancer.

Similarly, the association between passive smoking and lung cancer is based on other infections that cause chronic inflammation. A case-control study that investigated the role that Chlamydia Pneumoniae infection plays in the etiology or pathogenesis of lung cancer in combination with the interaction effects of smoking supported the association of passive smoking and lung cancer. ${ }^{9}$ The study showed that passive smokers with Chlamydia pneumoniae infections are at higher risks for lung cancer due to the toxic substances in tobacco smoke. The study revealed that the carcinogens available in cigarette or tobacco smoke in conjunction with Chlamydia Pneumoniae infections induce chronic inflammation due to the prolonged stimulation of inflammatory mediators in the lungs, leading to cell necrosis, mitosis, and apoptosis. Such action triggers the growth of lung cancer by altering cell replication, transcription, and repair of DNA damage. Another study showed that the reactive oxygen and nitrogen substances produced by smoking activate the transcription factor, NF-kB, which in turn enhances the expression of inflammatory genes and directly or indirectly causes the activation of inflammatory mediator production through the regulation of protein modification and protection degradations. ${ }^{10}$ As a consequence, the combination of Chlamydia Pneumoniae and toxic substances produced by tobacco smoke promotes lung cancer development due to higher levels of inflammatory factors that cause chronic inflammation in the lungs. Besides, exposure to second-hand tobacco smoke damages human cells in a way that rugger lung cancer process. The cancer-causing substances and poisons inhaled by nonsmokers cause cell damage in the lungs, which facilitates malignancy.

A study was conducted to determine the association of second-hand tobacco smoke and lung cancer based on histological types of adenocarcinoma, squamous cell 
carcinoma, small cell lung cancer, and large cell lung cancer. ${ }^{11}$ The study revealed that second-hand smoke has a significant impact on the histological lung cancer types. The study showed that second-hand smoke, when inhaled, causes cell damage in the respiratory systems, which in turn facilitates the development of lung cancer. Based on the histological types, the study disclosed that second-hand smoke or passive smoke among non-smokers is highly associated with all histological types, with non-smokers being at higher risk for adenocarcinoma. ${ }^{11}$ Another crosssectional study supported a strong association between passive smoking and lung cancer. The study assessed the association of second-hand smoke exposure and lung cancer among non-smokers aged between 16 years and 50 years in Telemark County in Norway. ${ }^{12}$ In their study, the researchers found that exposure to second-hand or passive smoke in non-smokers results in respiratory effects, including nocturnal dyspnoea and productive cough, which are the key predictors of lung cancer. The study indicated that frequent exposure to second-hand smoke increases the prevalence of chronic cough due to the chronic inflammation in the lungs, which triggers the pathogenesis of lung cancer. The study also found that increased exposure to second-hand smoke was associated with a reduction in lung functions in ever-smokers and never or non-smokers, with ever-smokers showing strong association because of frequent and high exposure to toxic substances of smoke, which causes lung inflammation that triggers the development of lung cancer. ${ }^{12}$ Similar findings were supported in a recent study among older Amish in the United States, which showed that high exposure to passive or second-hand smoke was associated with poor lung function, which is characterized by lower forced respiratory volume. ${ }^{13}$ Despite several studies indicate a strong association between passive smoking and lung cancer, very few studies show no link between the two. A prospective cohort study found a strong association between tobacco smoking and lung cancer but confirmed no association between second-hand smoking and lung cancer. ${ }^{18}$ The study indicated that in women who never smoked, exposure to second-hand smoking and other passive smoking had no significant impact on increasing the risk for lung cancer. ${ }^{18}$ The findings supported the clinical evidence of past studies that showed passive smoking did not have any significant association with an increased risk of developing lung cancer in both smokers and non-smokers. ${ }^{19,20}$ Although the studies supported no significant association between passive smoking and lung cancer, there are no current studies to prove such assumptions, which call for further research to validate such findings.

\section{CONCLUSION}

Lung cancer is one of the public health problems globally. Lung cancer has been among the leading cause of death and determining the main cause may help implement clinical interventions to reduce prevalence and mortality. Smoking is among the risk factors of lung cancer development. Cigarette smoking causes respiratory infections through chronic inflammation, which can trigger the development of lung cancer. Passive smoke or secondhand smoke has a significant impact on the development of lung cancer. The smoke has some toxic substances, including carcinogens, ammonia, carbon monoxide, and nitrosamines. These toxins, when exhaled by smokers, are inhaled by non-smokers, and this leads to chronic inflammation in the respiratory systems, leading to lung dysfunction, cell damage, and apoptosis that trigger the development of histological types of lung cancer. The main histological lung cancer types associated with passive smoke are squamous cell carcinoma, small cell lung cancer, large cell small cancer, and adenocarcinoma. The reviewed studies indicated a significant association between passive smoking and lung cancer. The studies supported that frequent exposure to environmental tobacco smoke or second-hand smoke increases the risk of developing lung cancer among non-smokers. Exposure at home and in public places, including parks, offices, schools, and restaurants, increases people's risks for lung cancer. The existing studies supported a strong association, which may help make the assumption that exposure to passive smoke by non-smokers and smokers enhances their potential to acquire lung cancer. However, some reviewed studies showed no association or a link between passive smoke and lung cancer. These studies supported that people exposed to second-hand smoke have no risks for lung cancer. These are past studies, and no current research has been conducted to prove such claims. However, the studies that showed strong associations outweigh those that oppose the association. This, therefore, provides the basis to conclude that passive smoking has significant impacts on lung cancer development. The studies provide substantial evidence that passive smoking causes lung cancer.

\section{Funding: No funding sources \\ Conflict of interest: None declared \\ Ethical approval: Not required}

\section{REFERENCES}

1. Furrukh M. Tobacco Smoking and Lung Cancer: Perception-changing facts. Sultan Qaboos Univ Med J. 2013;13(3):345-58.

2. Hymowitz N. Cigarette Smoking and Lung Cancer: Pediatric Roots. Lung Cancer Int. 2012;2012:790841.

3. Remen T, Pintos J, Abrahamowicz M, Siemiatycki J. Risk of lung cancer in relation to various metrics of smoking history: a case-control study in Montreal. BMC Cancer. 2018;18(1):1275.

4. Barta JA, Powell CA, Wisnivesky JP. Global Epidemiology of Lung Cancer. Ann Glob Health. 2019;85(1):8.

5. Naeem Z. Second-hand smoke - ignored implications. Int $\mathbf{J}$ Health Sci (Qassim). 2015;9(2):5-6.

6. Cao S, Yang C, Gan Y, Lu Z. The Health Effects of Passive Smoking: An Overview of Systematic 
Reviews Based on Observational Epidemiological Evidence. PLoS One. 2015;10(10):0139907.

7. Du Y, Cui X, Sidorenkov G, Groen HJM, Vliegenthart R, Heuvelmans MA, et al. Lung cancer occurrence attributable to passive smoking among never smokers in China: a systematic review and meta-analysis. Transl Lung Cancer Res. 2020;9(2):204-217.

8. Sun YQ, Chen Y, Langhammer A, Skorpen F, Wu C, Mai XM. Passive smoking in relation to lung cancer incidence and histologic types in Norwegian adults: the HUNT study. Eur Respir J. 2017;50(4):1700824.

9. Xu X, Liu Z, Xiong W, Qiu M, Kang S, Xu Q, et al. Combined and interaction effect of chlamydia pneumoniae infection and smoking on lung cancer: a case-control study in Southeast China. BMC Cancer. 2020;20(1):903.

10. Li T, He X, Chen Y. Mechanism of lung cancer and chronic obstructive pulmonary disease. J Central South University. Medical sciences. 2017;42(10):1212-6.

11. Kim CH, Lee YC, Hung RJ, McNallan SR, Cote ML, Lim WY, et al. Exposure to secondhand tobacco smoke and lung cancer by histological type: a pooled analysis of the International Lung Cancer Consortium (ILCCO). Int J Cancer. 2014;135(8):1918-30.

12. Fell AKM, Svendsen MV, Kim JL, Abrahamsen R, Henneberger PK, et al. Exposure to second-hand tobacco smoke and respiratory symptoms in nonsmoking adults: cross-sectional data from the general population of Telemark, Norway. BMC Public Health. 2018;18(1):843.

13. Reed RM, Dransfield MT, Eberlein M, Miller M, Netzer G, Pavlovich M, et al. Gender differences in first and secondhand smoke exposure, spirometric lung function and cardiometabolic health in the old order Amish: A novel population without female smoking. PLoS One. 2017;12(3):0174354.

14. Grapatsas K, Leivaditis V, Tsilogianni Z, Haussmann E, Kaplunov V, Dahm M, et al. Epidemiology, risk factors, symptomatology, TNM classification of Non Small Cell Lung Cancer. An overview while waiting the 8th TNM classification. Oncomedicine 2017;2:14-23.

15. Ridge CA, McErlean AM, Ginsberg MS Epidemiology of lung cancer. Semin Intervent Radiol. 2013;30(2):93-8.

16. Cao S, Yang C, Gan Y, Lu Z. The Health Effects of Passive Smoking: An Overview of Systematic Reviews Based on Observational Epidemiological Evidence. PLoS One. 2015;10(10):0139907.

17. Mu L, Liu L, Niu R, Zhao B, Shi J, Li Y, et al. Indoor air pollution and risk of lung cancer among Chinese female non-smokers. Cancer Causes Control. 2013;24(3):439-50.

18. Peres J. No clear link between passive smoking and lung cancer. J Natl Cancer Inst. 2013;105(24):1844-6.

19. Lee PN, Chamberlain J, Alderson MR. Relationship of passive smoking to risk of lung cancer and other smoking-associated diseases. $\mathrm{Br} \mathrm{J}$ Cancer. 1986;54(1):97-105.

20. Lee P. Passive smoking and lung cancer. Strength of evidence on passive smoking and lung cancer is overstated. BMJ. 1998;317(7154):346-7.

Cite this article as: Ambati SK. Association of passive smoking and lung cancer: Is there substantial evidence to prove the causation?. Int J Community Med Public Health 2021;8:2540-3. 